\title{
Ecological Interactions Between Metals and Microbes That Impact Bioremediation
}

Konopka, Allan E. Purdue University

\begin{abstract}
RESULTS TO DATE: Previous work showed the correlation between bacterial biomass, population
\end{abstract} structure and the amount of lead, chromium and aromatic compounds present along a $21.6 \mathrm{~m}$ transect in which the concentrations of both heavy metals ( $\mathrm{Pb}$ and $\mathrm{Cr}$ ) and aromatic compounds varied 2-3 orders of magnitude. This work suggested that (a) biomass level was better correlated to the level of biodegradable organic $C$ than the level of heavy metals, (b) microbial community composition differed between highly contaminated soils and uncontaminated ones, and (c) substantial microbial activity was found even in the highly contaminated soils. One confounding factor in these analyses was that the contaminated soils contained $\mathrm{Pb}, \mathrm{Cr}$, and aromatic hydrocarbons. Therefore, it was difficult to determine which factors were most important in the shifts of microbial community composition. Therefore, experiments were conducted in microcosms in which individual factors could be systematically varied. In this case, soils were used from the Seymour, IN site which had low levels of contamination, and the microbial community had had little chance to adapt to heavy metals or aromatic compounds. The objective of these experiments was to determine changes in soil bacterial community biomass, structure and activity after the addition of organic carbon (C) substrates (xylene or glucose) and heavy metals (Pb2+ or $\mathrm{Cr} 6+)$. The organic amendments were used to (a) provide the energy to drive changes in microbial community composition in response to heavy metal additions and (b) provide a means to monitor microbial activities in the microcosms (by analysis of carbon dioxide production in the systems). Two different organic amendments were used to represent a broadly used substrate (glucose) in which selective effects might primarily be due to heavy metals and an aromatic substrate (xylene) similar to the aromatic pollutants present at the site. In the case of xylene, selection would be on the basis of both capacity to catabolized xylene and resistance to heavy metals. Some general conclusions from these experiments are the following. The magnitude of the respiration and growth response was substantially less for xylene than for glucose. This may reflect differential reactions with the soil matrix that affect bioavailability. The lag in xylene degradation was due to a small initial population size of xylene degraders. Pb additions had a greater impact on xylene mineralization than on glucose. PLFA analysis suggests that metal additions have a greater overall effect on microbial community structure than does organic amendment. However, DGGE analyses illustrate that organic amendments may exert strong selective forces for specific catabolic types. Previous work demonstrated that microbial activity was present at metal levels that varied over 4 orders of magnitude, and that microbial community structure changed on the scale of meters as metal content varied. Some indications of this is that the culturable microbial communities in these soils contain many metal-sensitive bacteria, and that in situ analyses of microbial activity showed a very heterogeneous response to metal addition. Therefore, metal distribution and bioavailability may be patchy on much smaller scales. Arrays of samples were collected at 5, 15, and $50 \mathrm{~cm}$ distances. Three aggregates of $150 \mathrm{mg}$ from each sample were each subjected to sequential analysis of (a) microbial activity (14C-glucose mineralization), (b) community composition (DGGE analysis of 16S rDNA amplicons) and (c) extractable $\mathrm{Pb}$ and $\mathrm{Cr}$ content. The hypothesis was that microbial activity would be significantly related to metal content at this spatial scale. However, there was no correlation between metal contamination and activity level, for sets of samples at any spatial scale. There is a slight negative correlation between activity and metal at the 50 $\mathrm{cm}$ scale accounting for approximately $50 \%$ of the variation (R2=0.509). At the 5 and $15 \mathrm{~cm}$ scales there is no obvious trend between the level of metal contamination and microbial activity. Aggregates located < $1 \mathrm{~cm}$ from each other may differ in metal content by factors up to fivefold, and differ in microbial activity by $>1000$-fold. The community structure data indicated that there can be substantial differences in the microbial communities in aggregates located $<1 \mathrm{~cm}$ from each other. High metabolic activity was usually associated with complex community structure. The research has demonstrated substantial microbial activity in soils heavily contaminated with metals. However, both this work and the literature has indicated that some functional microbial groups (aromatic-degrading bacteria) might be especially susceptible to heavy metals. Therefore, it was attempted to cultivate and identify hydrocarbon degrading-metal resistant bacteria from a mixed waste site. Isolations were made from four soils contaminated with chromium (34- 
$87 \mathrm{mg} \mathrm{kg}-1$ ), lead (133-543 mg kg-1), and a mixture of aromatic compounds (from 0 to $>150 \mathrm{mg} \mathrm{kg}-1$ ). This phase of the project focused on the isolation of $\mathrm{Cr}$ resistant naphthalene degrading bacteria. Only a fraction of the culturable bacteria from a metal contaminated soil are resistant to that metal. Isolates were only obtained at $\mathrm{Cr}$ concentrations up to $5 \mathrm{mM}$. Microcosm enrichments containing minimal mineral broths with napthalene as a sole carbon source and $1 \mathrm{mM}$ potassium chromate were also used to isolate bacteria. After enrichments isolates tolerant to $20 \mathrm{mM} \mathrm{Cr}$ were obtained and naphthalene degraders were also obtained. However, $\mathrm{Cr}$ resistance and naphthalene degradation phenotypes did not cosegregate into single strains. Minimum inhibitory concentration (MIC) trials in liquid medium defined over 90 isolates capable of growing in $>1 \mathrm{mM} \mathrm{Cr}$, with over 20 isolates capable of growing in $20 \mathrm{mM} \mathrm{Cr}$. Genetic analysis based on the 16S rRNA gene indicates the majority of isolates belong to the same genus. Therefore, results suggest that heavy metal contamination may have more severe effects on specific functional groups of microbes. A more general analysis of the culturable chromium-resistant population in contaminated soils has also been carried out. A total of 119 chromate resistant isolates were obtained from two different metal contaminated soil samples (Seymour, Indiana and Cannelton, Michigan). The isolates? ability to tolerate different concentrations of chromium was determined and the isolates were characterized by BOX-PCR Fingerprinting. BOX-PCR Fingerprinting is a DNA typing technique that relies on the apparent genetic variability of the regions targeting the BOX motifs. The BOX-PCR patterns in conjunction with the morphological study revealed the presence of 22 different cultivable chromate resistant groups of isolates. In addition, a lead resistant Arthrobacter sp.VN23-1 wasislated that can transfer lead resistance by conjugation to a lead sensitive mutant. Subsequently a large plasmid in the size range of 80 to $100 \mathrm{~kb}$ was isolated from cultures of VN23-1 and cloned into E. coli. One of the clones, B23, which contains an insert of approximately $6.5 \mathrm{~kb}$ returned matches with homology to a number of heavy metal transport proteins including a lead efflux ATPase from Ralstonia metallidurans. Future work will focus on completing the sequence of the $6.5 \mathrm{~kb}$ insert and identifying the regions containing the genes for lead resistance.

DELIVERABLES: Beasley, F. C. and C. H. Nakatsu, Purdue University, West Lafayette, IN. 2002. Isolation and Characterization of Chromate Resistant Bacteria from Mixed Waste Contaminated Soil. Abstr. Annu. Mtg. Amer. Soc. Microbiol.

Shi, W., Bischoff, M., Turco, R. and Konopka, A. 2002. Association of microbial community composition and activity with $\mathrm{Pb}, \mathrm{Cr}$ and hydrocarbon contamination. Appl. Environm. Microbiol.,.in press

Shi, W., Bischoff, M., Turco, R. and Konopka, A. 2002. Long-term effects of chromium and lead upon the activity of soil microbial communities. Appl. Soil Ecology, in press.

Curtis, P., Nakatsu, CN, and Konopka, A. 2002. Aciduric Proteobacteria isolated from pH 3 soil. Archives of Microbiology, in press. 